\title{
Propuesta PARA el ANÁlisis del Diámetro Transversal: MÉtodo Barzallo An-Bar
}

\section{Proposal for the Analysis of The Transverse Diameter: Barzallo An-Bar Method}

\author{
Barzallo-Sardi Vinicio Egidio *1, Ordóñez-Pesántez María Gracia ${ }^{2}$. \\ ${ }^{1}$ Docente de la Facultad de Odontología - Universidad de Cuenca y Universidad de las Américas. \\ Miembro de la Sociedad Ecuatoriana de Ortodoncia. ${ }^{2}$ Odontóloga, Estudiante Especialidad de Ortodoncia - Universidad \\ Central del Ecuador. \\ Miembro de la Sociedad Ecuatoriana de Ortodoncia. \\ *vinicio.barzallo@ucuenca.edu.ec
}

\begin{abstract}
Resumen
El diagnóstico ortodóncico en la última década se ha potenciado con los avances tecnológicos, dejando de lado los métodos tradicionales de diagnóstico, como lo son el análisis de modelos, sobre todo cuando se trata del análisis transversal. Este trabajo busca brindar al clínico una opción sencilla en el diagnóstico del problema transversal, a través de modelos de yeso. Para sustentar esta propuesta se recolectaron datos de siete pacientes que fueron diagnosticados con compresión maxilar esqueletal, en edades comprendidas entre 10 y 14 años. Se tomaron datos de modelos de estudio en yeso extraduro, así como CBCT antes y después del tratamiento con MARPE. El análisis de UPenn se utilizó en la CBCT y el análisis de modelos se realizó midiendo desde el punto dentogingival derecho al izquierdo de los primeros molares permanentes superiores e inferiores, utilizando un calibrador Mitutoyo de $0.05 \mathrm{~mm}$ de exactitud. Se realizó la comparación de promedios de ambas medidas la cual demostró que no hay diferencia significativa y luego se hizo la correlación de medidas que demostró ser significativa en 5 de 7 medidas.

Esta propuesta de análisis de modelos (AN-BAR) puede ser útil para el clínico en la toma de decisión al momento de realizar una expansión maxilar.
\end{abstract}

Palabras clave: Análisis de modelos, tomografía computarizada con haz de cono (CBCT), deficiencia transversal.

\begin{abstract}
Orthodontic diagnosis in the last decade has been enhanced by technological advances, leaving aside traditional diagnostic methods such as model analysis, especially when it comes to a cross-sectional analysis. This work seeks to provide the clinician with a simple option in the diagnosis of the transverse problem, through plaster models. To support this proposal, data were collected from seven patients who were diagnosed with maxillary skeletal compression, aged between 10 and 14 years. Data were taken from study models in extradural plaster, as well as CBCT before and after treatment with MARPE. UPenn analysis was used in the CBCT and model analysis was performed by measuring from the right to left dentogingival point of the first upper and lower permanent molars, using a Mitutoyo caliper of $0.05 \mathrm{~mm}$ accuracy. A comparison of averages of both measurements was made which showed no significant difference and then the correlation of measurements was made which proved to be significant in 5 of 7 measurements.

This model analysis proposal (AN-BAR) may be useful for the clinician in making decisions when performing a maxillary expansion
\end{abstract}

Key words: Dental casts analysis, Cone Beam Computerized Tomography (CBCT), transverse deficiency.

\section{Introducción}

Los objetivos del ortodoncista están claramente establecidos con la finalidad de conseguir una relación oclusal funcional, armónica y estable, corrigiendo los problemas en los tres sentidos del espacio, se entiende que esto parte de un correcto diagnóstico; por lo tanto, realizar un diagnóstico que abarque estos tres objetivos es de vital importancia. Para ello se dispone de un sinnúmero de métodos para diagnosticar las relaciones entre los maxilares en la dimensiones vertical y sagital que son parte de la práctica diaria del ortodoncista. Sin embargo, referente al problema transversal existen varios métodos diagnósticos 
propuestos desde hace varios años, muchos de ellos con grandes limitaciones como es el hecho de no relacionar el maxilar superior con la mandíbula, por lo que no han sido aceptados dentro del método tradicional de diagnóstico en ortodoncia. $^{1-7}$

El presente trabajo abordará netamente el análisis de la dimensión transversal, con la propuesta de un método clínico de diagnóstico que brinda al profesional una opción más sencilla y a su vez efectiva al momento de diagnosticar un problema transversal.

El problema transversal es una anomalía esquelética, dental o combinada que clínicamente se manifiesta como mordida cruzada a nivel posterior cuando el origen de la maloclusión es producto de una base maxilar o mandibular desproporcionadamente ancha o estrecha con respecto a la otra, dando como resultado una discrepancia volumétrica transversal. $^{1,2}$ (Figura 1)

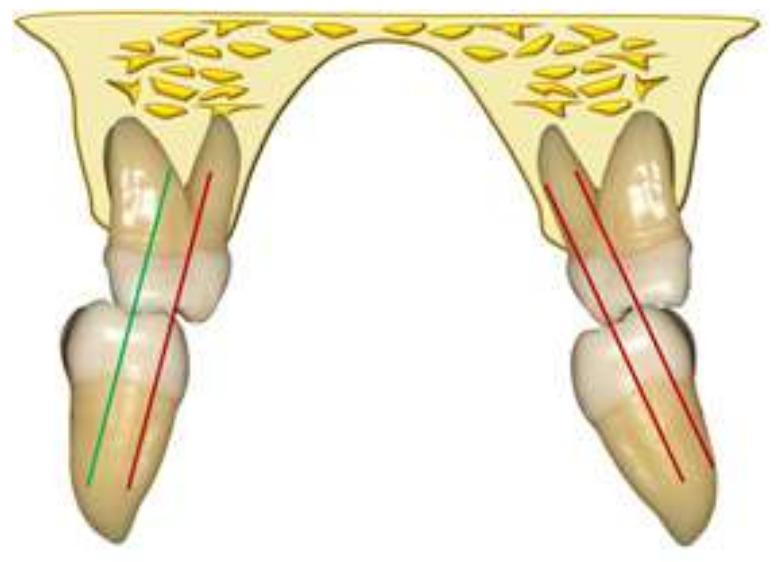

Fig. 1. Esquema discrepancia volumétrica transversal.Adaptada de Gregoret $^{1}$

Al presentar una deficiencia transversal es necesario entender que se ve alterada la relación entre las bases óseas y esta alteración a su vez causa que se vean afectadas estructuras importantes como son la articulación temporomandibular (ATM), musculatura orofacial, periodonto y vías aéreas; entonces la meta del ortodoncista debe ser proveer al paciente mediante los diferentes tratamientos una correcta relación entre los maxilares, proporcionando con esto una oclusión funcional lo más óptima posible. Así, el hecho de omitir el análisis transversal en el diagnóstico podría traer consecuencias importantes a la hora de hacer una expansión dentoalveolar como desplazamientos en céntrica por contactos bajos de cúspides molares causando daño articular, adelgazamiento de las corticales por colapso en la unidad periodontal, pérdida de soporte periodontal, recesiones gingivales y en casos severos dehiscencias y fenestraciones. ${ }^{3-5}$

Dentro de los métodos diagnósticos más populares utilizados para determinar la dimensión transversal están al análisis del cefalograma frontal de Ricketts-1969, que consiste en medir las dimensiones de los maxilares y compararlos con una tabla estandarizada con valores de norma., ${ }^{3,4,8}$ (Fig. 2)

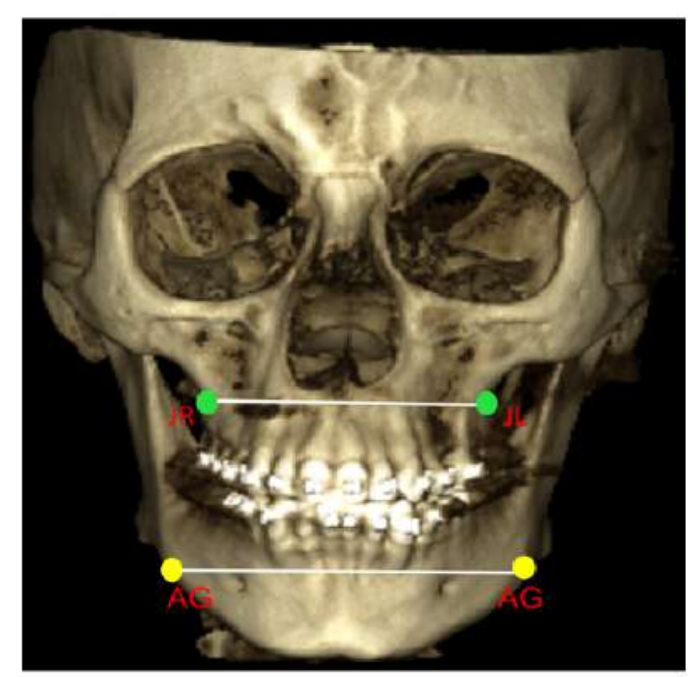

Tabla 1.-Valores normales de Ricketts

\begin{tabular}{|c|c|c|c|c|}
\hline EDAD & MAXILAR & MANDIBULA & DIFERENCIA & RADIO \\
\hline 9 & $62.0 \mathrm{~mm}$ & $76.0 \mathrm{~mm}$ & $14.0 \mathrm{~mm}$ & $81.6 \%$ \\
\hline 10 & $62.6 \mathrm{~mm}$ & $77.4 \mathrm{~mm}$ & $14.8 \mathrm{~mm}$ & $80.9 \%$ \\
\hline 11 & $63.2 \mathrm{~mm}$ & $78.8 \mathrm{~mm}$ & $15.6 \mathrm{~mm}$ & $80.2 \%$ \\
\hline 12 & $63.8 \mathrm{~mm}$ & $80.2 \mathrm{~mm}$ & $16.4 \mathrm{~mm}$ & $79.5 \%$ \\
\hline 13 & $64.4 \mathrm{~mm}$ & $81.6 \mathrm{~mm}$ & $17.2 \mathrm{~mm}$ & $78.9 \%$ \\
\hline 14 & $65.0 \mathrm{~mm}$ & $83.0 \mathrm{~mm}$ & $18.0 \mathrm{~mm}$ & $78.3 \%$ \\
\hline 15 & $65.6 \mathrm{~mm}$ & $84.4 \mathrm{~mm}$ & $18.8 \mathrm{~mm}$ & $77.7 \%$ \\
\hline 16 & $66.2 \mathrm{~mm}$ & $85.8 \mathrm{~mm}$ & $19.6 \mathrm{~mm}$ & $77.4 \%$ \\
\hline
\end{tabular}

Fig. 2. Trazado cefalograma frontal de Ricketts con su tabla de valores. Adaptada de Vanarsdall. ${ }^{4}$

Otro método para medir el diámetro transversal es el Elemento III de Andrews (Óptimo ancho de los maxilares), que propone que la mandíbula tiene un ancho óptimo, siendo que en caso de haber alguna discrepancia el problema es netamente maxilar, entonces menciona que la relación transversal entre los maxilares es correcta cuando la distancia que existe entre las fosas centrales de los primeros molares inferiores es igual a la distancia que existe entre las cúspides mesio-palatinas de los primeros molares superiores cuando los dientes se encuentran en Elemento I. 6,9,10 (Fig. 3)

Hoy en día el método más utilizado para el diagnóstico transversal es el análisis tomográfico (CBCT) propuesto por la Universidad de Pennsylvania-2010 (Análisis de UPenn) pues con el advenimiento del 3D se pueden obtener mediciones precisas sin mayor distorsión de estructuras, con más facilidad en la localización de puntos anatómicos. Esta propuesta diagnóstica consiste en determinar la cantidad de 


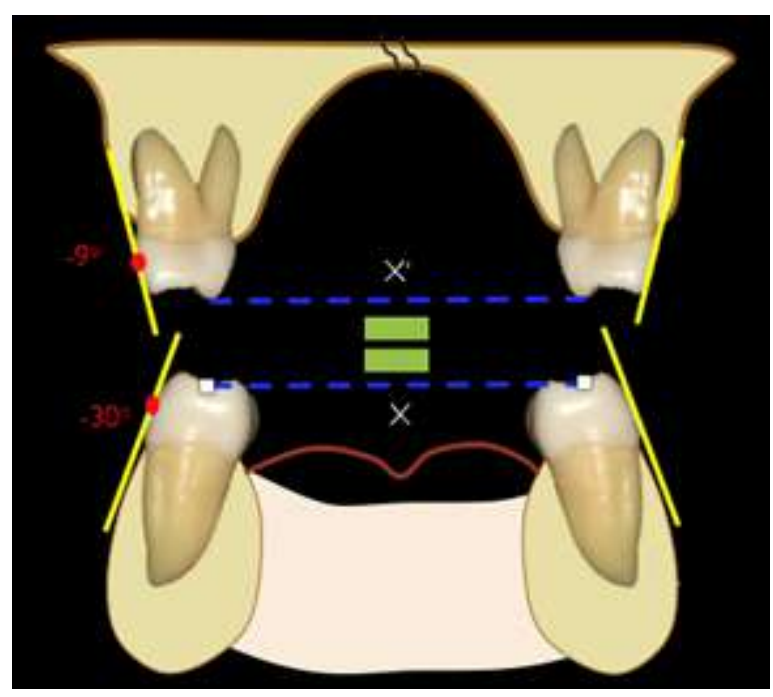

Fig. 3. Elemento III Andrews. Adaptada de la página Andrewsfoundation.org

discrepancia transversal entre los maxilares y se basa en el uso fácilmente discernible de puntos de referencia que representan el ancho de la base alveolar; para el maxilar superior se deben localizar los puntos jugales derecho e izquierdo propuestos por Ricketts, para la mandíbula es necesario ubicar los puntos a nivel de las furcas de los primeros molares inferiores; una vez localizados estos puntos se mide desde la cortical interna de las tablas externas de un lado al otro a nivel de estos, tanto en maxilar como mandíbula. El análisis del ancho del maxilar y mandibular es sencillo una vez que se han tomado las medidas de ambos maxilares. Al restar el ancho mandibular del ancho maxilar, se determina la diferencia entre estos. La diferencia aparentemente ideal para el ancho de los maxilares en pacientes maduros que utiliza el análisis Penn CBCT es de $5 \mathrm{~mm}$, es decir el maxilar debe ser $5 \mathrm{~mm}$ más grande que su mandíbula. ${ }^{3-9}$ (Fig. 4)

En base a estos análisis que vienen siendo los métodos más acertados de diagnóstico transversal y debido la dificultad en la ubicación de puntos cefalométricos en una radiografía frontal por la superposición de imágenes, a la subjetividad de evaluar un diente en base ósea y a que muchas veces los pacientes se rehúsan a invertir en un examen imagenológico que aún resulta costoso en nuestro medio, el presente estudio nace de la necesidad de implementar un método alternativo para diagnosticar la dimensión transversal, utilizando modelos de yeso que han sido una herramienta de diagnóstico desde hace muchos años en la práctica odontológica y propiamente en la ortodoncia. El objetivo del presente artículo es presentar la propuesta para el análisis del diámetro transversal: método Barzallo AN-BAR.

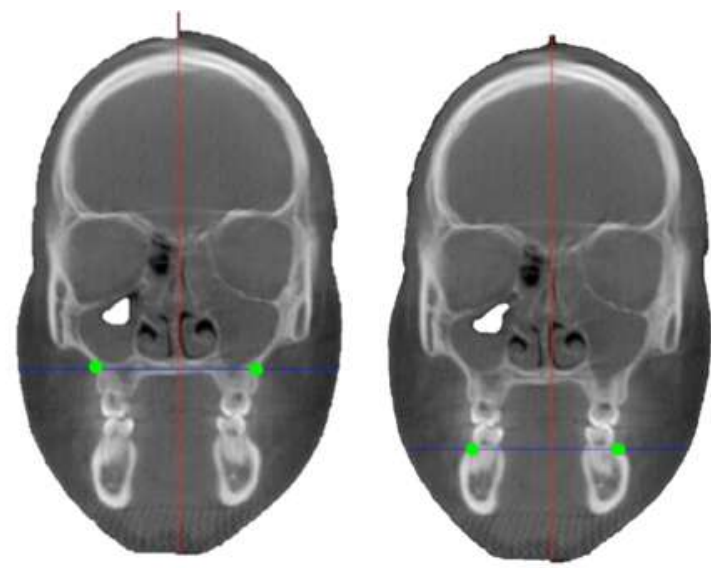

Fig. 4. Elemento III Andrews. Adaptada de la página Andrewsfoundation.org

\section{Estado del Arte}

Para respaldar esta propuesta se han compilado los datos de siete pacientes que fueron diagnosticados con compresión maxilar esqueletal, en edades comprendidas entre 10 y 14 años (12.29 años). Se tomaron modelos de estudio en yeso extraduro, así como CBCT antes y después del tratamiento con MARPE. Para el análisis de UPenn se utilizó la CBCT, sin embargo, en este estudio se hizo una pequeña modificación en la ubicación de los puntos maxilares, midiendo desde cortical interna de los primeros molares permanentes a nivel de la furca y no a nivel del punto Mx. En el maxilar inferior la medida fue hecha igual al análisis de UPenn. (Fig. 5 y 6). En el análisis de modelos propuesto por Barzallo (AN-BAR) se realizó midiendo por vestibular desde el punto dentogingival (fig. 7) derecho al izquierdo tanto de los primeros molares permanentes superiores como inferiores, utilizando un calibrador digital estandarizado Mitutoyo. (Mitutoyo Corporation cod. 500196-30) de 0.05mm de exactitud. (Fig. 8,9 y 10)

Con la información obtenida, se elaboró una base de datos en una hoja de cálculo de programa Excel Office 2013, luego se exportó a SPSS Ver 10. Para calcular los promedios de las mediciones, la comparación y la correlación entre las medidas en modelos y en CBCT mediante estadística no paramétrica (Tabla 2 y 3 ).

Al comparar los valores de las medidas entre datos de modelos y de CBCT, se encontró que la diferencia es estadísticamente no significativa en todas las medidas evaluadas (Tabla 2).

Luego al realizar las correlaciones entre las medidas de modelos y de CBCT, se encontró que la correlación es significativa en 5 de 7 medidas, solo en los datos iniciales del maxilar superior y en los datos de la diferencia del maxilar 


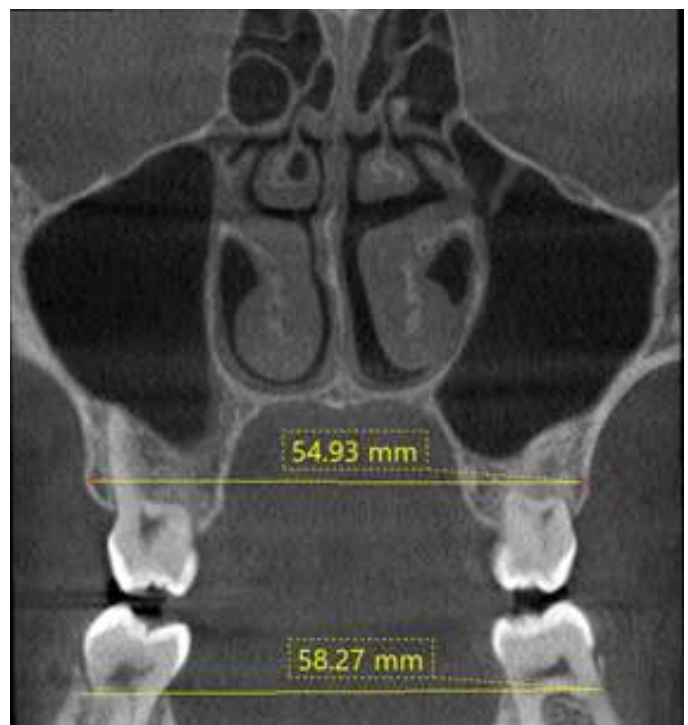

Fig. 5. Análisis CBCT antes.

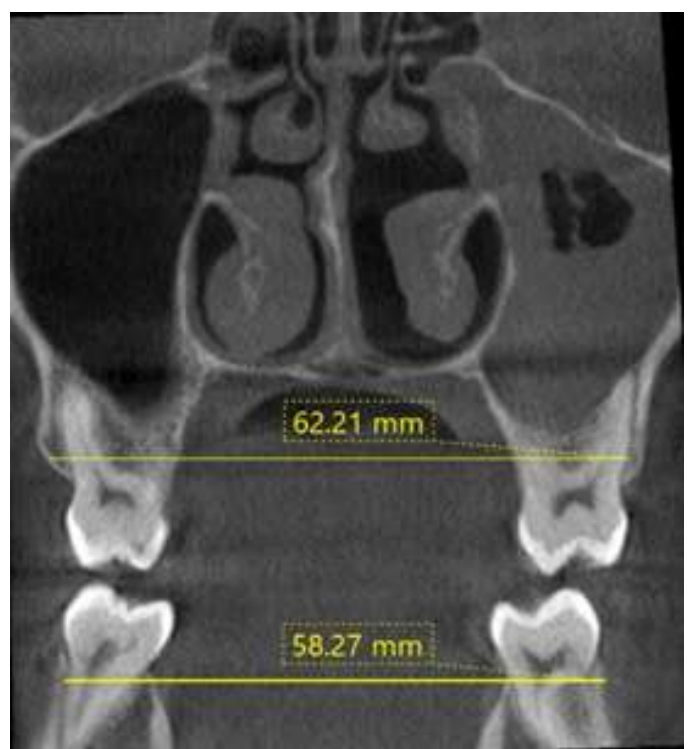

Fig. 6. Análisis CBCT después.

superior e inferior al final del tratamiento, resultaron tener una correlación significativa (Tabla 3).

El AN-BAR determina que el maxilar superior debe ser 5 mm más ancho que la mandíbula, lo que se correlaciona con el análisis de UPenn en CBCT.

\section{Discusión}

Está claramente definido que el diagnóstico de las maloclusiones se debe realizar en los tres sentidos del espacio, pues se entiende que la certeza de un diagnóstico lleva consigo un tratamiento adecuado.

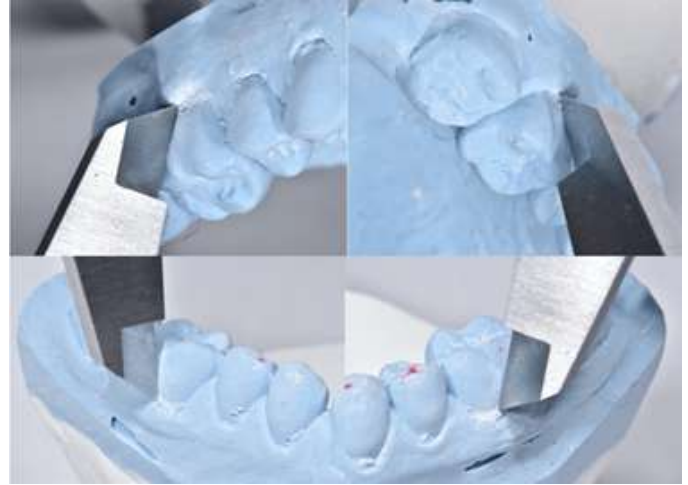

Fig. 7. Punto Dentogingival superior e inferior

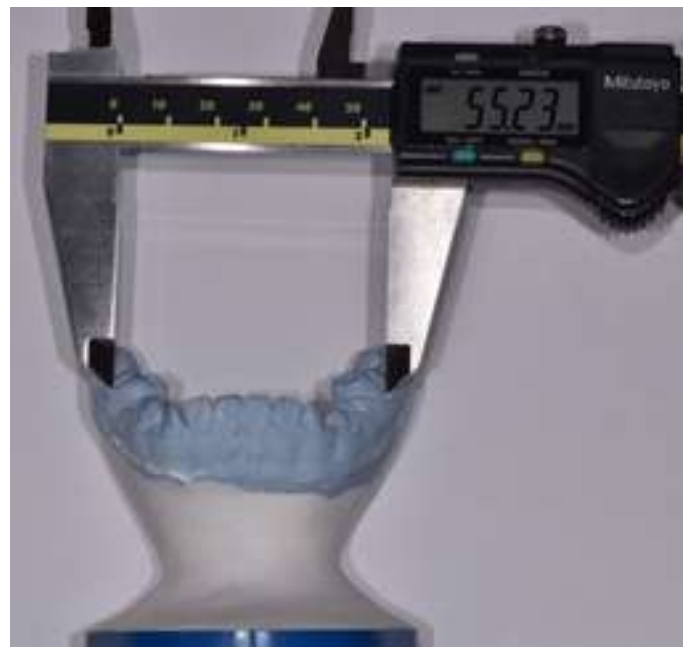

Fig. 8. Modelo inferior

Al respecto Tamburrino y cols. ${ }^{3}$ en su publicación presentan tres métodos de diagnóstico transversal sin categorizarlos, uno que utiliza la radiografía postero anterior basado en el análisis del cefalograma frontal de Ricketts, un segundo análisis que emplea modelos de estudio propuesto por Andrews, conocido como Elemento III y un tercer análisis propuesto por la Universidad de Pennsylvania que usa tomografía computarizada cone-beam (CBCT), la propuesta de los autores en este artículo es netamente proporcionar a los lectores estas herramientas de diagnóstico para que se incorpore dentro de la práctica diaria al análisis transversal sin importar cuál de ellos se elija, pero siempre teniendo en cuenta los objetivos en mente.

Por otro lado, Miner y cols. ${ }^{11,12}$ realizaron un estudio para determinar la sensibilidad y especificidad del análisis tomográfico (CBCT) para determinar los anchos maxilares, ellos establecieron que este método tiene un alto porcentaje de sensibilidad y especificidad.

Sawchuck y cols. ${ }^{13}$ en una revisión sistemática de métodos 
Tabla 2: Comparación entre las medidas de modelos y de CBCT.

\begin{tabular}{|c|c|c|c|c|c|c|c|c|c|c|c|c|c|c|}
\hline & $\begin{array}{l}\text { INICIAL INF } \\
\text { CBCT }\end{array}$ & $\begin{array}{l}\text { INICIAL INF } \\
\text { MODELOS }\end{array}$ & $\begin{array}{l}\text { INICIAL SUP } \\
\text { CBCT }\end{array}$ & $\begin{array}{l}\text { INICIAL SUP } \\
\text { MODELOS }\end{array}$ & $\begin{array}{l}\text { IDEALL } \\
\text { MAXULAR } \\
\text { CBCT }\end{array}$ & $\begin{array}{l}\text { IDEAL } \\
\text { MANLAR } \\
\text { MODELOS }\end{array}$ & $\begin{array}{l}\text { DIF CON EL } \\
\text { IDEAL CBCT }\end{array}$ & $\begin{array}{c}\text { DIF CON EL } \\
\text { IDEAL } \\
\text { MODELOS }\end{array}$ & $\begin{array}{c}\text { DIFERENCIA } \\
\text { SUPERIOR E } \\
\text { INFERIOR } \\
\text { INICIAL } \\
\text { CBCT }\end{array}$ & $\begin{array}{l}\text { DIFERENCIA } \\
\text { SUPERIOR E } \\
\text { INFERIOR } \\
\text { INICIAL } \\
\text { MODELLOS }\end{array}$ & $\begin{array}{l}\text { FINAL } \\
\text { SUPERIOR } \\
\text { CBCT }\end{array}$ & $\begin{array}{c}\text { ANAL } \\
\text { SUPERIOR } \\
\text { MODELOS }\end{array}$ & $\begin{array}{l}\text { DIFERENCIA } \\
\text { SUPERIORE } \\
\text { INFERIOR } \\
\text { FINAL CBCT }\end{array}$ & $\begin{array}{c}\text { DIFERENCIA } \\
\text { SUPERIOR E } \\
\text { INFERIOR } \\
\text { FNAL } \\
\text { MODELOS }\end{array}$ \\
\hline Media & 54,79 & 54,78 & 54,14 & 53,65 & 59,79 & 59,78 & 5,65 & 6,13 & $-0,65$ & $-1,13$ & 59,76 & 59,6 & 4,97 & 4,82 \\
\hline $\begin{array}{l}\text { Mnimo } \\
\text { Maximo }\end{array}$ & $\begin{array}{c}50,1 \\
58,27\end{array}$ & $\begin{array}{c}51,6 \\
58,62\end{array}$ & $\begin{array}{l}51,3 \\
57,3\end{array}$ & $\begin{array}{l}48,98 \\
58,26\end{array}$ & $\begin{array}{c}55,1 \\
63.27\end{array}$ & $\begin{array}{c}56,6 \\
63,62\end{array}$ & $\begin{array}{r}2,3 \\
9,13\end{array}$ & $\begin{array}{l}1,93 \\
9,05\end{array}$ & $\begin{array}{c}-4,13 \\
2.7\end{array}$ & $\begin{array}{r}-4,05 \\
3,07\end{array}$ & $\begin{array}{l}55,2 \\
63,4\end{array}$ & $\begin{array}{l}55,3 \\
64,1\end{array}$ & $\begin{array}{c}3,94 \\
6,4\end{array}$ & $\begin{array}{c}2,98 \\
5,7\end{array}$ \\
\hline SIG & \multicolumn{2}{|c|}{$p=1,00$} & \multicolumn{2}{|c|}{$p=0.87$} & \multicolumn{2}{|c|}{$p=1,00$} & \multicolumn{2}{|c|}{$p=0,612$} & \multicolumn{2}{|c|}{$p=0,612$} & \multicolumn{2}{|c|}{$p=0.866$} & \multicolumn{2}{|c|}{$p=0,866$} \\
\hline
\end{tabular}

Tabla 3: Correlación entre las medidas de modelos y de CBCT

\begin{tabular}{|c|c|c|c|c|c|c|c|}
\hline \multicolumn{8}{|c|}{ Correlaciones entre las medidas en Modelos y CBCT } \\
\hline $\begin{array}{l}\text { Rho de } \\
\text { Spearman }\end{array}$ & $\begin{array}{l}\text { INICIAL } \\
\text { INFERIOR }\end{array}$ & $\begin{array}{l}\text { INICLAL } \\
\text { SUPERIOR }\end{array}$ & $\begin{array}{l}\text { IDEAL } \\
\text { MAXILAR }\end{array}$ & $\begin{array}{l}\text { DIF.CON } \\
\text { EL IDEAL }\end{array}$ & $\begin{array}{l}\text { DIF. SUP. E } \\
\text { INFERIOR } \\
\text { INICIAL }\end{array}$ & $\begin{array}{l}\text { FINAL } \\
\text { SUPERIOR }\end{array}$ & $\begin{array}{l}\text { DIF. SUP. E } \\
\text { INFERIOR } \\
\text { FINAL }\end{array}$ \\
\hline $\begin{array}{l}\text { Coef. de } \\
\text { correlación }\end{array}$ & $0,821^{\circ}$ & 0.500 & $0,821^{\circ}$ & $0.786^{\circ}$ & $0,786^{\circ}$ & $0,893^{-\prime}$ & 0,107 \\
\hline Sig. (bilateral) & 0,023 & 0,253 & 0,023 & 0,036 & 0,036 & 0,007 & 0,819 \\
\hline $\mathbf{N}$ & 7 & 7 & 7 & 7 & 7 & 7 & 7 \\
\hline
\end{tabular}

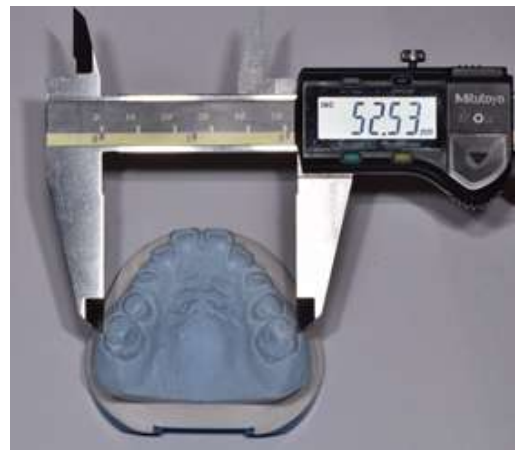

Fig. 9. Modelo superior antes de MARPE

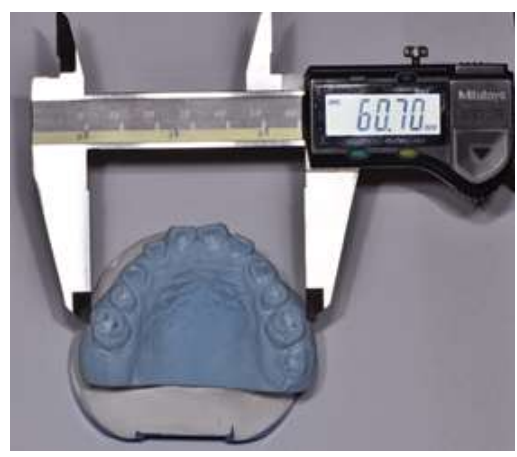

Fig. 10. Modelo superior después de MARPE

para evaluar la deficiencia transversal esqueletal y dental concluyeron que en análisis tomográfico es el de mayor confianza para evaluar las deficiencias transversales del maxilar. En cuanto al análisis de modelos, Andrews $\mathrm{L}^{6-10}$ en el Elemento III menciona que el ancho maxilar es óptimo cuando la distancia entre las cúspides palatinas de los primeros molares superiores coincide con las fosas centrales de los primeros molares inferiores siempre que los dientes se encuentren en Elemento I, es decir que estén en hueso basal y las inclinaciones y angulaciones sean las ideales. Una limitación de este análisis consiste en el hecho de que los pacientes que llegan a la consulta, la gran mayoría no se encuentra en elemento I, entonces este análisis ya no sería aplicable en esos casos; al respecto de esta situación los autores mencionan que existe la forma de predecir el ancho de los maxilares, pero sigue siendo una opción subjetiva porque estaría más a criterio del clínico que de algo medible o reproducible.

Un estudio de Guerra $\mathrm{A}^{14}$ que analizó la sensibilidad y especificidad entre tres métodos diagnósticos que incluyó el análisis póstero anterior (PA) de Ricketts, análisis en CBCT y el análisis de Hayes en modelos digitales concluyó que tanto los análisis en CBCT como el de modelos poseen más sensibilidad diagnóstica que el PA de Ricketts. A diferencia de lo que se propone en este método diagnóstico, el análisis de modelos de Hayes mide a nivel de las crestas alveolares para el diagnóstico transversal, se mide a través del ancho bucolingual/palatino tanto en la arcada superior como inferior a nivel de la unión amelocementaria (dónde se encuentra la cresta alveolar), se marca la mitad de esta medida en el lado derecho como el izquierdo; en el maxilar se mide la distancia de centro a centro a nivel de las cúspides mesiales de los primero molares y en la mandíbula a nivel de las fosas centrales en la mitad previamente marcada. ${ }^{15}$ Una dificultad de este análisis es la determinación real de la cresta alveolar, debido a que muchos pacientes pueden presentar pérdida de soporte periodontal y el nivel de la cresta ósea varía en un gran porcentaje en estos casos, entonces viene a ser un análisis con cierto grado de subjetividad.

En la presente propuesta se utilizó el análisis tomográfico 
(CBCT) como referencia puesto que como se revisó anteriormente, es el método de mayor confianza al momento de medir diámetro transversal.

Se observa en base a los resultados, que existe una correlación bastante significativa entre esta propuesta de diagnóstico en base a modelos con el análisis en CBCT de la UPenn. Una fortaleza de esta propuesta radica en el hecho de no necesitar un examen complementario adicional como lo es una tomografía (CBCT), pues con los registros habituales como son los modelos de estudio se puede diagnosticar una deficiencia transversal, además de ser un método bastante cómodo y rápido de efectuar. Al tomar como referencia la unión dentogingival, que en los modelos de yeso se presenta incluso como un tope, facilita al clínico para que al momento de realizar la medición no exista mayor distorsión de las medidas obtenidas, pues es un punto bastante objetivo. No obstante, es necesario mencionar que, como todos, este análisis también tiene sus limitaciones, como son el hecho que no podría aplicarse en caso de que los primeros molares estén: ausentes, con grandes rotaciones, inclinaciones vestíbulolinguales muy marcadas y grandes recesiones gingivales. Sin 11 embargo, es una alternativa perfectamente extrapolable a la gran mayoría de la población que acude a consulta en busca de un tratamiento ortodóncico.

Finalmente, por los resultados expuestos en este trabajo en cuanto a la correlación que existe entre esta propuesta de análisis de modelos y el análisis tomográfico de la UPenn, se puede inferir que ambos métodos son equivalentes al momento de diagnosticar la dimensión transversal.

\section{Conclusiones}

- Existe una correlación estadísticamente significativa entre los valores del CBCT y el análisis de modelos ANBAR propuesto en este trabajo, donde el maxilar superior debe ser $5 \mathrm{~mm}$ más ancho que la mandíbula, por lo que es aplicable para el diagnóstico de la dimensión transversal.

- Aplicar el AN-BAR simplifica el procedimiento y recursos a la hora de diagnosticar un problema transversal, favoreciendo al clínico en la toma de decisión al momento conocer cuántos milímetros necesita de expansión.

Agradecimiento: Al Dr. Ebingen Villavicencio Caparó por la asesoría estadística de esta investigación.

Conflicto de intereses Los autores declaran no tener ningún conflicto de interés.

\section{Referencias}

1 Gregoret J. Ortodoncia y Cirugía Ortognática. Diagnóstico y Planificación. 2da edicion. Caracas: Amolca; 2014. p 151155.

2 Interlandi S. Ortodoncia Interlandi. 1ra edicion. Brasil: Artes Médicas; 2002.

3 Tamburrino R, Boucher N, Vanarsdall R, Secchi A. The Transverse Dimension: Diagnosis and relevance to Funtional Occlusion. RWISO Journal. Sept, 2010.
4 Vanarsdall RL Jr. Transverse dimension and long-term stability. Semin Orthod. 1999; 5: 171-180.

5 Vanarsdall RL, White RP Jr. Three-dimensional analysis for skeletal problems. Int J Adult Orthodon Orthognath Surg. 1994; 9: 159.

6 Andrews L. The six keys to normal occlusion. Am J Orthod Dentofacial Orthop. 1972; 62 (3): 296-309.

7 Lee KM, Hwang HS, Cho JH. Comparison of transverse analysis between posteroanterior cephalogram and conebeam computed tomography. Angle Orthodontics, 2014; 84 (4).

8 Ricketts RM. Perspectives in the clinical application of cephalometrics, the first fifty years. Angle Orthod. 1981;51: 115-150.

9 Andrews L, Andrews W. Andrews analysis. In: Sylabus of the Andrews Orthodontic Philosophy. Six Elements Course Manual: 2001.

10 Andrews L. The 6-elements orthodontic philosophy: Treatment goals, classification, and rules for treating. Am J Orthod Dentofacial Orthop 2015; 148: 883-7

11 Miner R, Qabandi S, Rogali P, Will L. Cone Beam computed tomografy transverse analysis. Part 2: Measures of performance. Am J Orthod Dentofacial Orthop. 2015; 148 (2).

12 Miner R, Qabandi S, Rogali P, Will L. Cone Beam computed tomografy transverse analysis. Part 1: Normative data. Am J Orthod Dentofacial Orthop. Sept, 2012; 142 (3).

13 Sawchuk D, Currie K, Lagravere M, Palomo JM, FloresMir C. Diagnostic methods for assessing maxillary skeletal and dental transverse deficiencies: A systematic review. The Korean Journal of Orthodontics. 2016; 46 (5): 331-345.

14 Guerra GA et al. Sensitivity and specificity of a radiographic, tomographic and digital model analysis Revista Mexicana de Ortodoncia. 2018; 6 (1): 26-32.

15 Hayes J. In search of improved skeletal transverse diagnosis-Part2: A new measurement technique used on 114 consecutive untreated patients. Orthodontic Practice. 2010; 1(4).

16 Hayes J. In search of improved skeletal transverse diagnosis. Part 1: Traditional measurement techniques. Orthodontic Practice. 2009; 1(3). 